\title{
POTENCIAUDAD DE SIETE ESPECIES FORESTALES PARA LA RESTAURACIÓN DESDE EL NIVEL DEL MAR HASTA 3.000 M EN VERACRUZ, MÉXICO
}

\section{Juan Alba-Landa, Lilia Mendizábal-Hernández* Virginia Rebolledo-Camacho}

InSTITUTO de Genética Forestal

Universidad VeracruZana (MÉXico)

*Imendizabal@uv.mx

\section{INTRODUCCIÓN}

México, con una diversidad de especies forestales del orden de tres mil no conoce más de $20 \mathrm{de}$ acuerdo al manejo de la diversidad contenida en cada una de ellas. Desconoce por lo tanto su potencial de adaptación, desarrollo o conservación en sitios diferentes o en su rango de distribución natural, situación por la cual resulta de primer orden implementar estudios de investigación que específicamente aporten conocimiento a interrogantes fundamentales como son movimiento de fuentes y límite mínimo y máximo altitudinal en el que se puede desarrollar cada una sin perder productividad. Sólo de esta manera se puede planear el desarrollo y conservación de los recursos naturales de manera interactuante con el desarrollo y demanda de la sociedad para poder asumir una postura real en lo teórico y metodológico que nos permita realizar un manejo integral, entendido como la cohabitación equilibrada de todas las especies vivas de un ecosistema.

La situación geográfica del estado de Veracruz en México que presenta paisajes desde el nivel del mar hasta los $5.700 \mathrm{~m}$, y por otro lado la característica de que en una línea no mayor de $80 \mathrm{Km}$ cubre el rango altitudinal mencionado, hace posible se presente variación clinal, variación por aislamiento geográfico, variación por interacción y variación de exposición topográfica entre otras. Siendo la variación la materia prima para el mejoramiento genético forestal (Nienstaedt, 1990), resulta de vital importancia probar la diversidad contenida en cada especie, población o familia, en sitios determinados con el objeto de explorar movimientos no sólo de especies sino de posibles fuentes para sitios específicos.

Esto se logra a través del establecimiento de pruebas de procedencias y de procedencia/progenie, de tal forma que a través de réplicas de cada una de las diferentes especies en distintos pisos altitudinales podamos incursionar en la determinación del tamaño de sitio, tomando en cuenta que cada individuo de una población es un paquete único e irrepetible de genes y en consecuencia su habilidad de interacción con el ambiente es diferente, considerado un sitio como un espacio físico en el que se puede mover una fuente sin que pierda considerablemente su productividad. 
La diversidad altitudinal que se da en el área de estudio y consecuentemente sus efectos ambientales resultan atractivos para investigaciones como la presente, donde el objetivo a alcanzar fue el establecer una estrategia de elección de fuentes parentales de siete especies forestales en el estado de Veracruz, México.

\section{MATERIAL Y MÉTODOS}

Para el presente trabajo se utilizaron siete especies componentes del bosque tropical, del bosque mesófilo y del bosque de altura correspondientes a una distribución del nivel del mar a los $4.200 \mathrm{~m}$.

Para cada una de las especies elegidas, después de conocer su rango de distribución natural, se muestrearon - de acuerdo a las características deseablesindividuos componentes de cada una de las poblaciones de interés siguiendo la metodología recomendada por Patiño y Garzón (1976), a los cuales les fue colectada la semilla, germinadas en vivero y establecidas en campo en un diseño de bloques completos al azar en donde fueron evaluadas las poblaciones y las familias componentes de cada uno de los individuos de cada población en su caso o progenie proveniente de cada uno de los individuos colectados en bosque natural y establecidos por especie y rango de acuerdo a lo siguiente:
A cada una de estas pruebas les fue evaluado el diámetro y la altura como indicadores de la interacción de cada componente individual en cada bloque en los sitios establecidos.

\section{RESULTADOS}

Con la finalidad de garantizar el abastecimiento de semilla de calidad y de origen conocido es que se localizaron y delimitaron 5 rodales semilleros, uno de Pinus greggii Engelm. ubicado en Carrizal Chiquito del municipio de Zacualpan, otro de Pinus oocarpa Schiede ubicado en el municipio de San Pedro Soteapan y tres de Pinus teocote Schiede \& Deppe, el primero ubicado en Mixquiapan en el municipio de Jalacingo, otro en la localidad de Maguelles en el municipio de Maltrata y el último en la localidad de Palo Bendito en el municipio de Huayacocotla, teniéndose como resultado la selección de 25 árboles de Pinus greggii Engelm. en Carrizal Chiquito, 30 árboles de Pinus oocarpa Schiede en San Pedro Soteapan y 90 árboles de Pinus teocote Schiede \& Deppe (30 árboles en cada rodal).

También se cuenta con un área semillera de Pinus patula Schlect et Cham. ubicada en el ejido Ingenio El Rosario del municipio de Xico, Veracruz la cual abastece de semilla de calidad para los programas de reforestación de la zona del Cofre de Perote y de la

\begin{tabular}{|l|c|l|}
\hline ESPECIE & RANGO DE DISTRIBUCION ALTITUDINAL (msnm) & AUTOR \\
\hline Cederla odorata & $0-800$ & \\
\hline Liquidambar styraciflua & $900-1600$ & Sosa (1978) \\
\hline Pinus caribaea & $0-1500$ & G. van Wyk (2002) \\
\hline Pinus greggii & $1300-2700$ & G. van Wyk (2002) \\
\hline Pinus hartweggi & $3000-3700$ & Perry (1991) \\
\cline { 2 - 3 } & $2815-4000$ & Eguiluz (1985) \\
\cline { 2 - 3 } & $3000-4000$ & C. Rodríguez-Franco (2002) \\
\hline \multirow{3}{*}{ Pinus maximinoi } & $800-1500$ & Perry (1991) \\
\cline { 2 - 3 } & $700-2400$ & B.I. Nioka (2002) \\
\hline \multirow{3}{*}{ Pinus patula } & $1500-3100$ & Dvorak y Donahue (1992) \\
\cline { 2 - 3 } & $2100-2800$ & Perry (1991) \\
\cline { 2 - 3 } & $1800-2700$ & Look (1977) \\
\cline { 2 - 3 } & $1800-3000$ & Vela (1980) \\
\cline { 2 - 3 } & $1250-3300$ & B.I. Nioka (2002) \\
\cline { 2 - 3 } & $1500-2500$ & Donahue (1991) \\
\hline Pinus teocote & $1300-3000$ & Codríguez-Franco (2002) \\
\hline
\end{tabular}




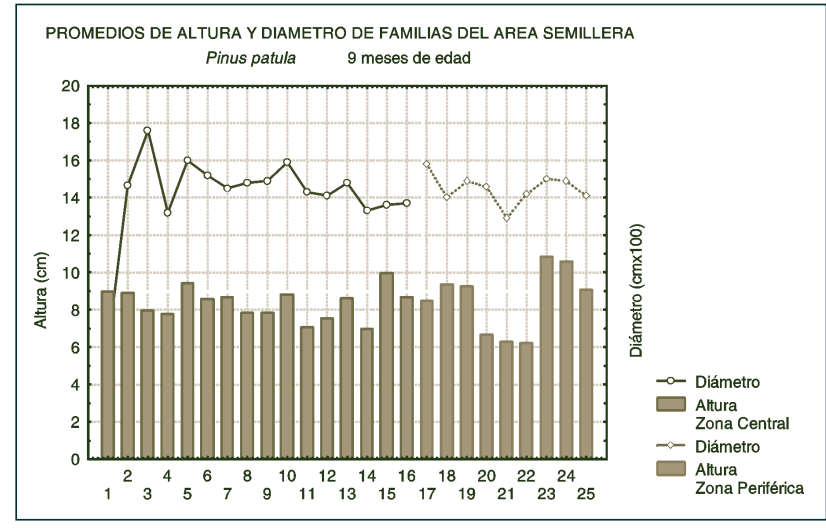

Figura 1. Comparación del crecimiento de la progenie de las familias de la zona central del área semillera Ing. Raúl Martínez, Ingenio El Rosario Veracruz, México.

cual ha sido posible identificar las familias que proveen de los mejores crecimientos a su progenie para dicha zona (Figura 1), al comparar el crecimiento promedio de la progenie de las familias de las zonas central y periférica (Rebolledo, 1995).

\section{VARIACIÓN EN EL TAMAÑO DE FRU- TOS DE CEDRELA ODORATA}

Se evaluó la variación en el ancho y largo de frutos de diez familias procedentes de la población natural de la Antigua Veracruz (Figura 2), así como el potencial de producción de semillas, determinándose que el tamaño de los frutos no influye en el potencial de semillas (Rodríguez y col. 2001). Con la semilla se estableció una plantación en el municipio de Emiliano Zapata, Veracruz a una altitud de $400 \mathrm{msnm}$ para evaluar el crecimiento de estas mismas familias. Los datos existentes revelan una variación proporcional entre el tamaño de frutos y semilla con el desarrollo de las plántulas establecidas en campo, por lo que se espero seguir comprobando que para un sitio es necesario buscar la fuente adecuada no solo dentro de las poblaciones o procedencias sino dentro de familias e incluso de individuos componentes de una familia.

En el presente estudio se ha establecido en el estado de Veracruz desde 1990 a la fecha 22 plantaciones experimentales que abarcan 7 especies forestales del Estado. De estas plantaciones se ha tenido el seguimiento en el crecimiento de las familias y

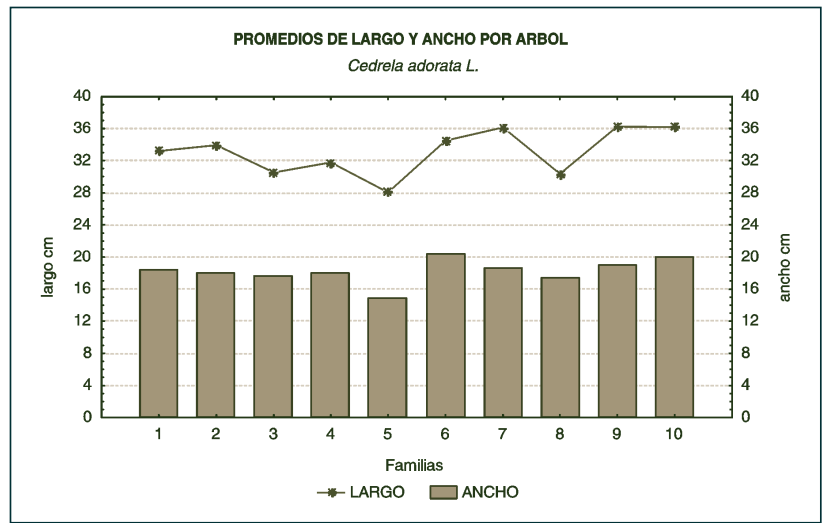

Figura 2. Comparación del tamaño de fruto de diez familias de Cedrela odorata.

procedencias que los conforman y cuyos resultados se resumen a continuación.

\section{a) Ensayos de procedencias y proce- dencias-progenie}

Estos ensayos se han establecido para evaluar y determinar la variación en el crecimiento de las especies en cada uno de los sitios del estado de Veracruz que se presenta en distintas poblaciones de la misma especie a través de su rango de distribución natural, así como la variación entre sitios y entre individuos, esto al evaluar la progenie en un mismo sitio de cuyos resultados podamos decidir la mejor procedencia, la mejor familia y el mejor individuo de cada una de ellas para el sitio en donde fue establecido y poder determinar así la estrategia que permita producir mas en menor tiempo y espacio.

En un ensayo con Pinus hartwegii se están probando 7 poblaciones procedentes del Estado de México (4) y del Distrito Federal (3), aun cuando no se ha establecido la plantación, se cuenta con la evaluación del crecimiento a los 10 meses de edad bajo condiciones de vivero, dando como resultado que seis de las procedencias presentaron promedios de altura que variaron de entre 4 a $5 \mathrm{~cm}$ y diámetros de entre 1 y $2 \mathrm{~cm}$, únicamente una de las procedencias tuvo alturas muy inferiores pero diámetro mayores a las demás procedencias, con la cual ha sido posible identificar la existencia de variación entre procedencias. 


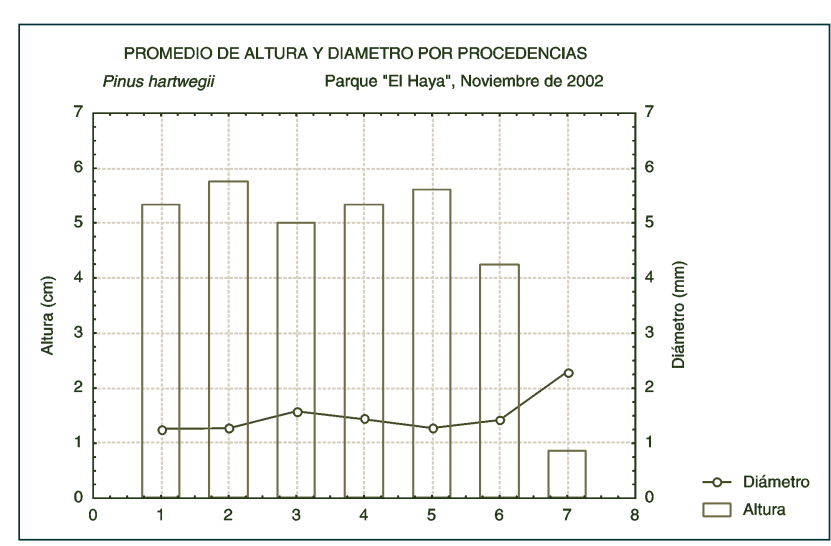

Figura 3. Comparación del crecimiento de siete procedencias de Pinus hartwegii.

Pinus caribaea especie importante por ser una alternativa para el trópico húmedo que además representa la por su distribución desde casi el nivel del mar hasta cerca de los 900 msnm. La plantación está establecida en el rancho Santa Isabel, localizado en el municipio de Santiago Tuxtla, Veracruz a una altitud de $210 \mathrm{msnm}$. En ella se encuentran representadas 5 poblaciones procedentes de Honduras. Los resultado de crecimiento en altura y diámetro a los 3 años de edad se presenta en la figura 4; observándose que la procedencia de Los Limones difiere considerablemente de las demás, alcanzando los mayores crecimientos e incluso es factible observar que dos de las familias evaluadas son superiores en altura y diámetro promedio (Alba-Landa y col. 1998).

Pinus maximinoi cubre ampliamente un gradiente altitudinal y condiciones ecológicas subtropicales con una buena productividad en comparación con otras

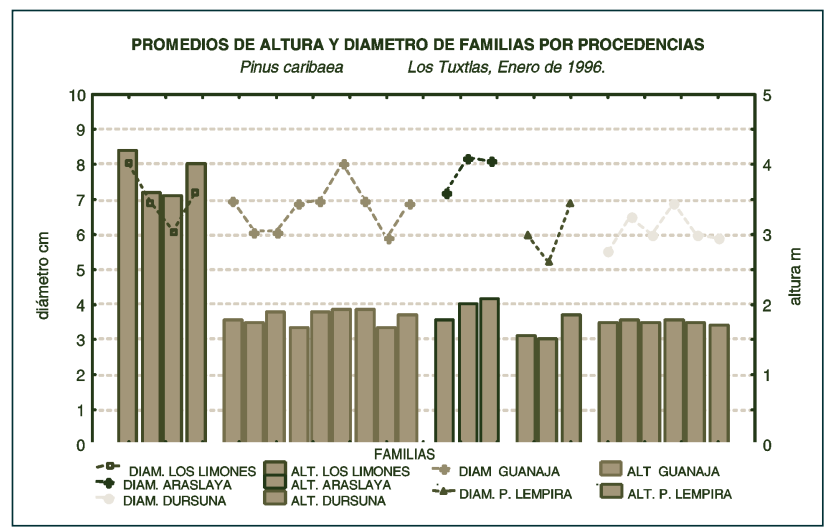

Figura 4. Comparación del crecimiento promedio de las familias de cinco procedencias de Pinus caribaea.

especies como $P$. oocarpa. Se establecieron dos plantaciones en el municipio de Xalapa, Ver. a una altitud de $1.220 \mathrm{~m}$ (Parque El Haya) y $1.400 \mathrm{~m}$ (Anexo Parque Fco. Xavier Clavijero), las cuales están situadas en condiciones similares, difiriendo únicamente en la exposición, en ambas se están probando 3 poblaciones procedentes de Honduras, una de Guatemala y 4 de México, evaluándose un total de 36 familias de las cuales ha sido posible comprobar que mismas familias en condiciones ambientales varían en cuanto al crecimiento por lo que se reafirma la necesidad de seleccionar no solo procedencias sino también familias e incluso individuos para cada sitio de plantación y que el tamaño de éste es también muy particular (Márquez, 1999).

De Pinus greggi se cuenta con material genético procedente de 3 poblaciones de México, evaluándose un total de 21 familias en una plantación ubicada en el Arenal, municipio de Coatepec, Veracruz

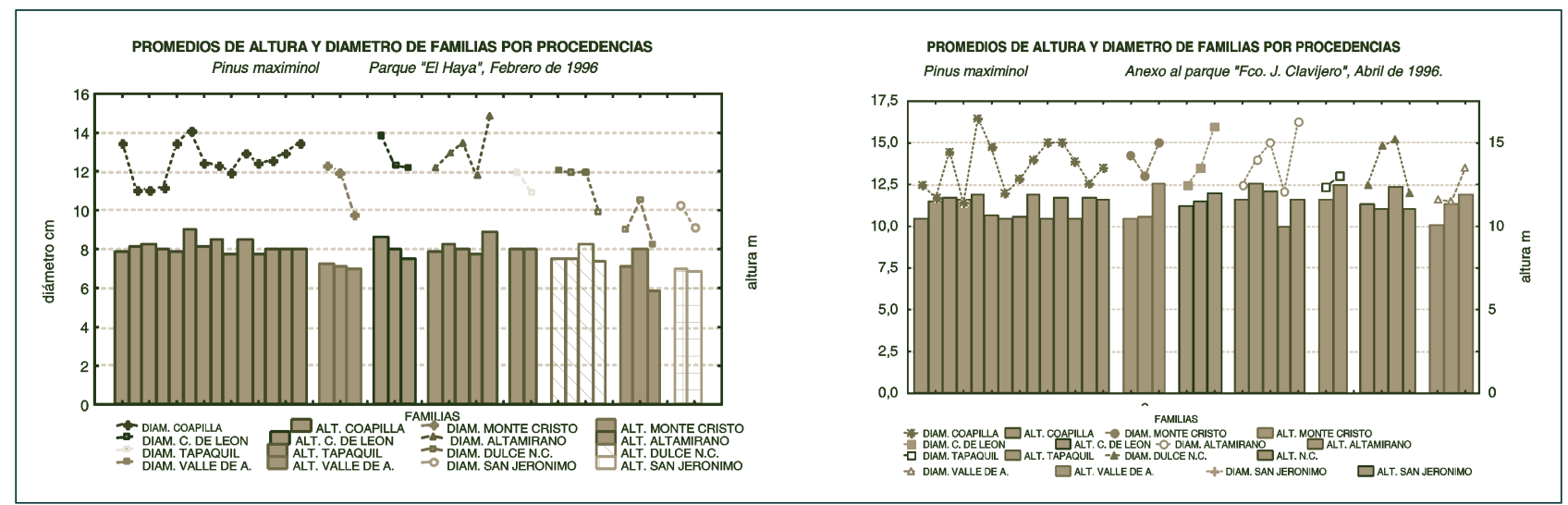

Figura 5. Comparación del crecimiento promedio de las familias de ocho procedencias de Pinus maximinoi. 


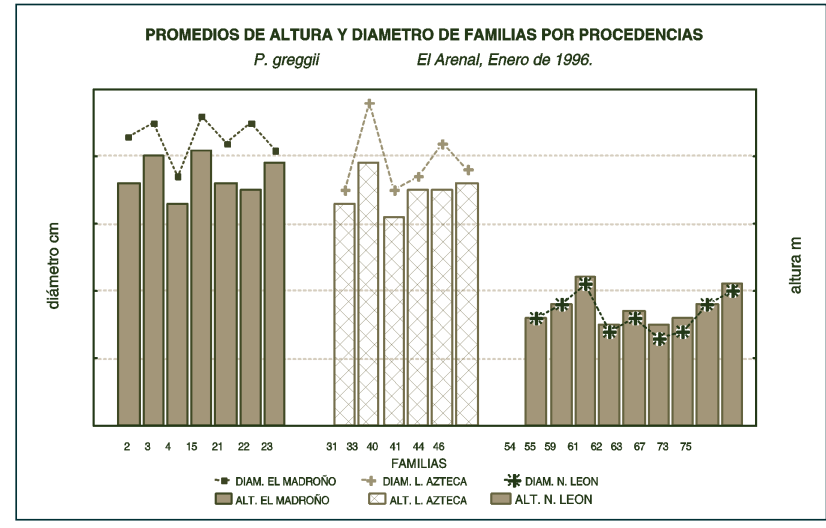

Figura 6. Comparación del crecimiento promedio de las familias de tres procedencias de Pinus greggii.

a $1200 \mathrm{msnm}$. De acuerdo a la figura 6 es clara la variación en el crecimiento entre y dentro de procedencias, comprobándose que para dicho sitio, las procedencias El Madroño y Laguna Azteca presentan los mejores crecimientos (Alba, 1996).

Liquidambar styraciflua es una de las principales especies de latifoliadas de la zona templada del estado de Veracruz, por lo cual se logro conjuntar en una plantación material genético procedente de 10 poblaciones naturales de México, Guatemala, Honduras y Nicaragua y una de la zona local, ésta se localiza a $1420 \mathrm{msnm}$ en el municipio de Xalapa, Veracruz. Al evaluar y compara el crecimiento promedio por procedencia fue factible corroborar la existencia de tres procedencias con alturas y diámetros superiores a las demás $(\mathrm{C}, \mathrm{F}$ y $\mathrm{K})$, incluso a la local (X) (Falcón, 2000).

De Pinus teocote se establecieron tres plantaciones experimentales localizadas en la zona central del

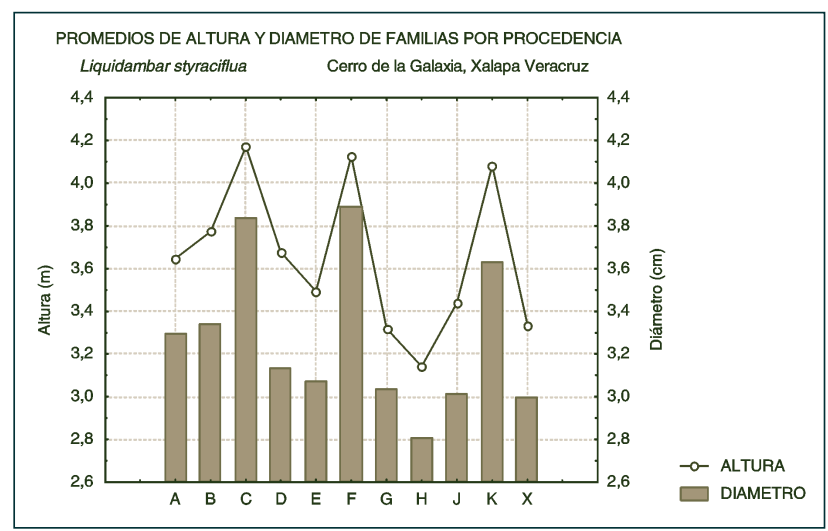

Figura 7. Comparación del crecimiento promedio de las procedencias de Liquidambar styraciflua.

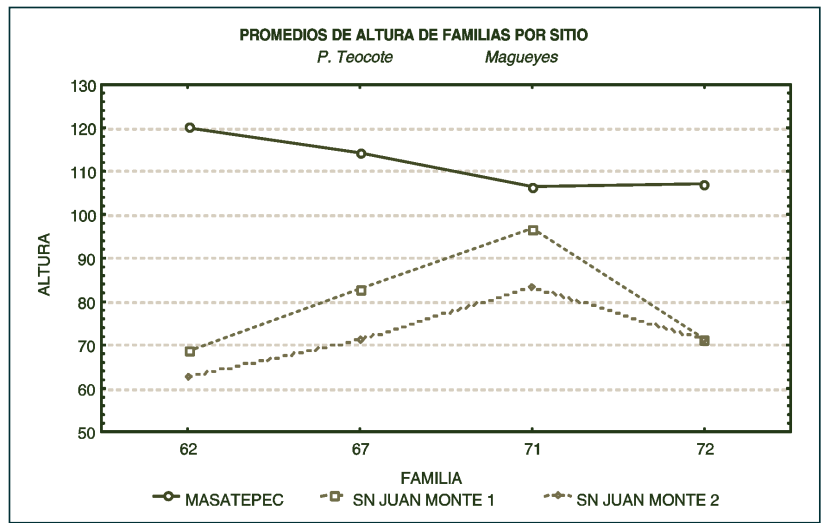

Figura 8. Comparación del crecimiento promedio de 4 familias de la procedencias de Magueyes en los tres sitios de plantación.

estado de Veracruz, a altitudes de 2.200 (Mazatepec), 2.600 (San Juan del Monte 1) y 2.860 (San Juan del Monte 2) en las cuales se están evaluando el crecimiento promedio en altura de la progenie de $14 \mathrm{fa}$ milias procedentes de tres poblaciones naturales de dicha especie. Como ejemplo se presenta el crecimiento promedio para uno de los sitios, observándose un comportamiento diferente de las familias en cada uno de los sitios (figura 8).

\section{b) Ensayos de Progenie}

Ejemplares de Pinus patula procedente de Veracruz fueron sometidos a un proceso de selección y cruzas en Sudáfrica, después del cual semilla de varias familias de un huerto semillero de segunda generación de selección regresaron para ser evaluadas en cuanto a su desarrollo nuevamente en el estado. Con esta semilla se establecieron 5 plantaciones en igual número de sitios y a partir del cual se ha logrado observar la variación existente en cuanto al desarrollo de las mismas progenies en condiciones diferentes, principalmente en cuanto a la altitud de los sitios (figura 9).

\section{CONCLUSIONES}

Con los resultados hasta ahora obtenidos es posible establecer un esquema de regeneración utilizando los espacios físicos donde se puede mover una fuente proveniente de una especie dependiendo de la capacidad de interacción al sitio que presentan poblaciones, familias e individuos; según el gradiente 


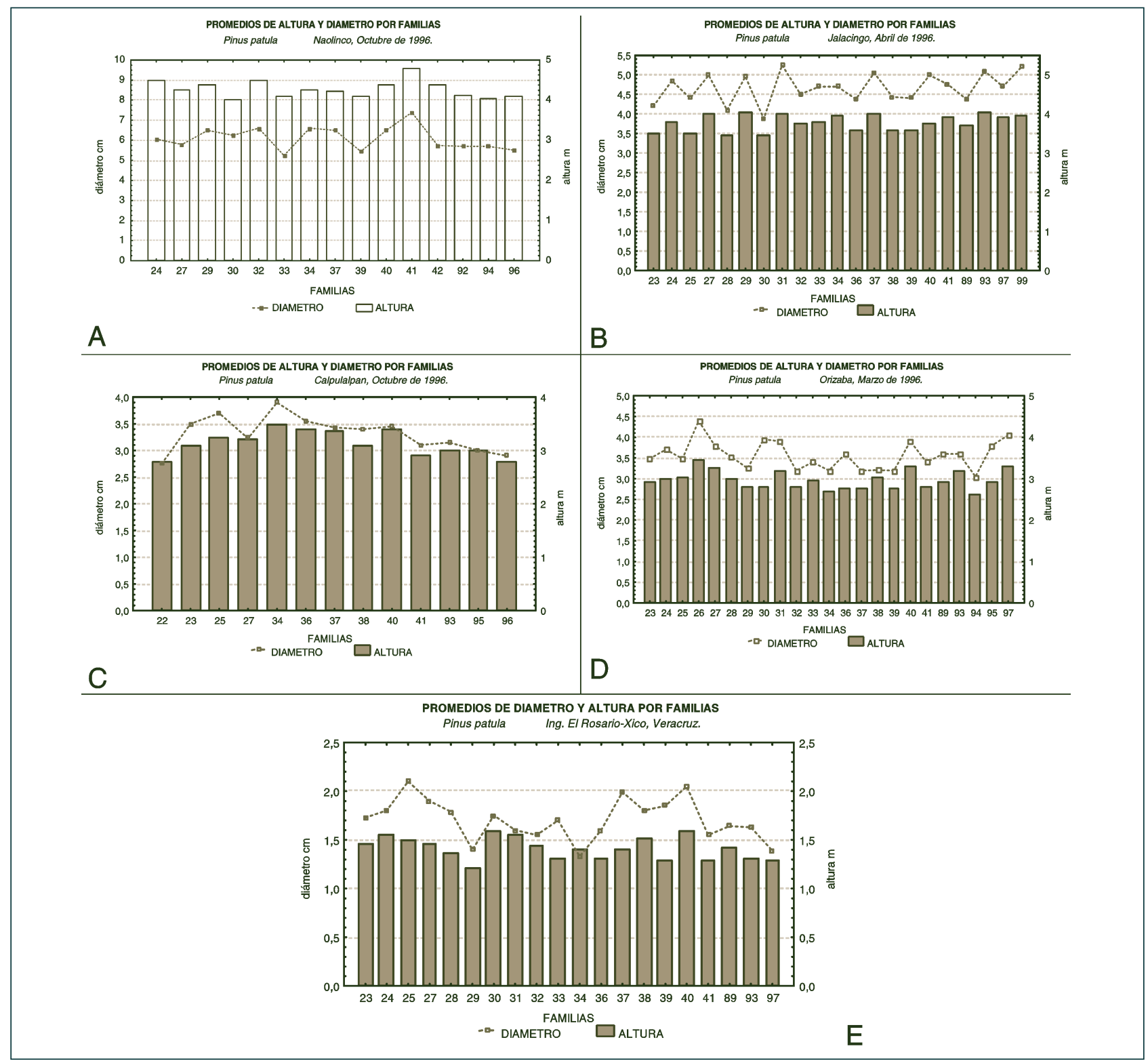

Figura 9. Comparación del crecimiento promedio de la progenie de las diferentes familias de Pinus patula procedentes de huertos semilleros de segunda generación de selección en diferentes sitios.

altitudinal y componentes de cada especie que a continuación se detalla:

- Pinus caribaea desde el nivel del mar hasta los 600 msnm.

- Cedrela odorata desde el nivel del mara hasta los 800 msnm.

- Pinus maximinoi de los 900 a los 1400 msnm.

- Pinus greggii de los 1200 a los $1700 \mathrm{msnm}$.

- Liquidambar styraciflua de los 1400 a los 1900 msnm.

- Pinus patula de los 1700 a los 2600 msnm.

- Pinus teocote de los 2000 a los 3000 msnm.
Para el movimiento de familias usando como ejemplo $P$. teocote se percibe que en un tamaño de sitio que varia de 2200 a $2860 \mathrm{msnm}$, la productividad es considerablemente diferente para mismas familias.

\section{LITERATURA CITADA}

ALBA-LANDA, J., MENDIZÁBAL-HERNÁNDEZ, L. Y REBOLLEDO-CAMACHO, V. 1998. Pinus caribaea Mor. var hondurensis Barr. y Golf. como alternativa para plantaciones tropicales. Foresta Veracruzana 1(1):33.36. 
ALBA LANDA, J. 1996. Mejoramiento genético forestal en el estado de Veracruz. Tesis de Maestría. Instituto de Genética Forestal, Universidad Veracruzana. Xalapa, Veracruz México. 80 p.

DVORAK, W.S. y DONAHUE, J.K. 1993. Reseña de investigaciones de la Cooperativa CAMCORE 1980-1992. Traduc. Meneses, J. Departamento Forestal, Colegio de Recursos Forestales, Universidad Estatal de Carolina del Norte. Raleigh, Carolina del Norte, USA. 94 p.

Eguiluz P. T. 1985. Descripción botánica de los pinos mexicanos. División de Ciencias Forestales, U.A.CH. Chapingo, México.

FALCON SANTIAGO, C. 2000. Evaluación de la variación de un ensayo de procedencias de Liquidambar styraciflua L. en Xalapa, Ver, México. Tesis de Maestría. Instituto de Genética Forestal, Universidad Veracruzana. Xalapa, Veracruz México. 48 p.

LOOCK, E.E.M. 1977. The pines of Mexico and British Honduras. Bulletin 35. Union of South Africa Dept. for Pretoria. 261 p.

MÁRQUEZ RAMÍREZ, J. 1999. Estudio comparativo del crecimiento de procedencias y familias de Pinus maximinoi Moore en dos ensayos con diferente exposición en el municipio de Xalapa, Veracruz, México. Tesis de Maestría. Instituto de Genética Forestal, Universidad Veracruzana. Xalapa, Veracruz México. 48 p.

MENDIZÁBAL, H. L. 1995. Evaluación de la progenie de dos huertos semilleros de Pinus patula Schlect. et Cham. provenientes de Sudáfrica, en el estado de Veracruz, México. Tesis profesional, Facultad de Biología. Universidad Veracruzana, Xalapa, Veracruz. 76 p.

NIENSTAEDT, H. 1990. Importancia de la variación natural. En: Mejoramiento genético y plantaciones forestales. Memoria. Editores: Teobaldo Eguiluz Piedra y Antonio Plancarte Barrera. Lomas de San Juan, Chapingo, México. pp. 16-23.

NYOKA, B.I. 2002. Pinus maximinoi. In: Pines of silvicultural importance. Complied form the Forestry Compendium, CAB International. CABI Publishing. Pp. 219-222.

NYOKA, B.I. 2002. Pinus patula. In: Pines of silvicultural importance. Complied form the Forestry Compendium, CAB International. CABI Publishing. Pp. 303-315.
PATIÑO, V.G. y GARZÓN, J.C.R. 1976. Manual para el establecimiento de ensayos de procedencia. Bol. Div. Inst. nac. Invest. For. 43. México. $61 \mathrm{p}$.

PERRY, J.T. 1991. The pines of Mexico and Central America. Timber Press. Portland, Oregon, USA. 231 p.

REBOLLEDO CAMACHO, V. 1995. Evaluación en vivero de la progenie de un área semillero de Pinus patula Schlect. Et Cham. localizada en el ejido Ingenio El Rosario municipio de Xico, Veracruz. Tesis de licenciatura en biología. Facultad de Biología, Universidad Veracruzana. Xalapa, Veracruz México. 60 p.

REBOLLEDO C. V. 1997. Estudio comparativo de cuatro ensayos de progenie de Pinus patula Schlect, et Cham. en el estado de Veracruz, México. Tesis de Maestría en Ecología Forestal. Centro de Genética Forestal. Universidad Veracruzana. Xalapa, Ver. 41 p.

RODRÍGUEZ RIVAS, G.; MÁRQUEZ RAMÍREZ, J. Y REBOLLEDO CAMACHO, V. 2001. Determinación del potencial y eficiencia de producción de semillas de Cedrela odorata L. y su relación con caracteres morfológicos de frutos. Foresta Veracruzana 3(1): 23-26.

RODRÍGUEZ-FRANCO, C. 2002. Pinus hartwegii. In: Pines of silvicultural importance. Complied form the Forestry Compendium, CAB International. CABI Publishing. Pp. 160-164.

RODRÍGUEZ-FRANCO, C. 2002. Pinus teocote. In: Pines of silvicultural importance. Complied form the Forestry Compendium, CAB International. CABI Publishing. Pp. 494-497.

SOSA, V. 1978. Hamamelidaceae. En: Flora de Veracruz. INIREB. Fascículo No. 1. 6 páginas.

VAN WYK, G. 2002. Pinus caribaea. In: Pines of silvicultural importance. Complied form the Forestry Compendium, CAB International. CABI Publishing. Pp. 38-50.

VAN WYK, G. 2002. Pinus greggii. In: Pines of silvicultural importance. Complied form the Forestry Compendium, CAB International. CABI Publishing. Pp. 144-146.

VELA, G. L. 1980. Contribución a la ecología de Pinus patula Schlecht et Cham. INIF México. Public. Esp. No. 19. 109 p. 